\title{
Register nominum ad Vol. XXXII
}

Arcieri, G. P. 255 (B)

Azan Cano, L., v. Castellanos, A.

Balaguer-Vintró, I., v. Torner-Soler,

M. Baroldi, G., v. Mantero, O. Barrow, D. W. 191 (B) Bige, G., v. Kenedi, I. Bøe, J.;

Humerfelt, S., and Weder-

vang, F. 328 (B) Bolt, W.; Forssmann, W. and Rink,

H. 187 (B) Braun, K., v. Eliakim, M.

Calò, A. 37

Calviño, J. M., v. Castellanos, A.

Cannata, D., and Narbone, N. B. 329

Carlier, J., v. Geubelle, F. Castellanos, jr., A.; Azan Cano, L.

and Calviño J. M. 212 Cignolini, P. 64 (B)

Denolin, H., v. Lequime J. Desruelles, J., v. Legrand, R. Dibsy, A. J., v. Donzelot E.

Donzelot, E.; Heim de Balsac, R.;

Rager, C. R. et Dibsy, A. J. 109 Dreyfuss, F.; Zlotnick, A. and

Menczel, J. 220 Dubouloz, P. 193 Duchosal, P. W., et Moret, P. 129

Eliakim, M.; Izak, G. and Braun, K.

177

388 Register nominum

Feruglio, F. S. et Migheli, B. 317 Forssmann, W., v. Bolt, W.

Gardberg, M., 384 (B) Gernez-Rieux, Ch., v. Legrand, R. Geubelle, F., et CarlieГ, J. 295

Grettve, J., and Johansson B. 374 Gross, D., 302

Harvald, B., v. Kemp, T.

Hauge, M., v. Kemp, T.

Hedlund, S., v. Nylin, G.

Heim de Balsac, R., v. Donzelot, E.

Heinecker, R. 191 (B)

Humerfelt, S., v. Bøe, J.

Izak, G., v. Eliakim, M.

Johansson, B., v. Grettve, J. Jonsson, B. J. 155

Kaufmann, E. und Staemmler, M.

128 (B), 187 (B) Kemp, T.; Hauge, M. and Harvald,

B.. 61 (B) Kenedi, I., und Bige, G. 278 Kopsch, F. 256 (B) Kornel, L. 101 Kubicek, F. 257

Laszt, L.; Meier, R. et Müller, A.

192 (B) Legrand, R.; Gernez-Rieux, Ch.;

Desruelles, J.; Voisin, C. et Pau-

chant, M. 21 Lequime, J. et Denolin, H. 65 Lottenbach, K., und Scharf, R. 10 Lüthy, E. 291

Mainzer, F. 362

Mantero, O.; Baroldi, G. and 
Scomazzoni, G. 48 Marchesani, O. und Sautter, H.

189 (B) Meier, R., v. Laszt, L.

ad Vol. XXXII

Menczel, J., v. Dreyfuss, F. Migheli, B., v. Feruglio, F. S. Morató-Portell, J. M., v. Torner-

Soler, M. Moret, P., v. Duchosal, P. W. Müller, A., v. Laszt, L.

Narbone, N. B., v. Cannata, D. Nylin, G., and Hedlund, S. 1

Olivier, C., 256 (B)

Pariscenti, P., 383 (B) Pauchant, M., v. Legrand, R. Pederson, A. 326 (B)

Rager, C. R., v. Donzelot, E. Rager, G. R. 61 (B) Rink, H., v. Bolt, W.

Saint-Jacques, A. J., v. Schelling,

J.-L. Sauerteig, E. 85 Sautter, H., v. Marchesani, O. Scharf, R., v. Lottenbach, K. Schelling, J.-L., et Saint-Jacques,

A. J. 119 Schennetten, F. P. N., 63 (B) Scomazzoni, G., v. Mantero, O. Siegenthaler, W., 161 Siguier, F. 256 (B) Staemmler, M., v. Kaufmann, E. Sulser, U.-J. 231

Torner-Soler, M.; Balaguer-Vintró,

I. et Morató-Portell, J. M. 355 Trethewie, E. R. 345

Voisin, C., v. Legrand, R. Völker, R. 127 (B)

Wedervang, F., v. Bøe, J. White, P. D. 191 (B)

Zlotnick, A., v. Dreyfuss, F. Zuckermann, R. 62 (B) 